\title{
DIPTERES TORRENTICOLES PEU CONNUS : III. - LES ATHERICIDAE DU SUD DE LA FRANCE (REGGIME ALIMENTAIRE DES LARVES : ASPECT QUALITATIF) \\ [Brachycera, Orthorrhapha]
}

\author{
par A. G. B. Thomas ${ }^{1}$.
}

\begin{abstract}
L'étude expérimentale en eau courante et en aquariums clos du régime alimentaire d'Atherix ibis révèle une polyphagie essentiellement constituée de larves d'insectes torrenticoles à cuticule mince : Ephéméroptères Baetidae et Heptageniidae, Trichoptères Philopotamidae et Diptères Limoniidae et Chironomidae. Le temps de succion (digestion extraorale) dépend du rapport de la taille de la proie à celle du prédateur et varie d'une quinzaine de minutes à deux heures environ. Chez les larves de plus de $4 \mathrm{~mm}$, l'appareil buccal est efficace pour des proies d'une large gamme de tailles. L'intensité de la prédation exercée $\grave{a}$ un moment donné par des larves d'A. ibis sur une population de Chironomidae âgés est une fonction linéaire de la longueur des prédateurs entre 5 et $15 \mathrm{~mm}$.

Le cannibalisme est presque inexistant lorsque les larves ne sont pas privées de nourriture. Les individus âgés peuvent supporter un jeûne de plus de 6 mois entre 10 et $15^{\circ} \mathrm{C}$.

Quelques observations ont également été faites sur le régime alimentaire d'Atrichops crassipes.
\end{abstract}

\section{Poorly known torrential Diptera : \\ III. Athericidae of the south of France (qualitative aspects of the food or the larvae) (Brachycera, Orthorrhapha).}

An experimental study in both flowing water and a closed aquarium showed that larvae of Atherix ibis are polyphagous, feeding on the larvae of torrential insects with thin chitin: Ephemeroptera (Baëtidae, Heptageniidae), Trichoptera (Philopotamidae), Diptera (Limoniidae, Chironomidae). Digestion is extra-oral and the suction time is a function of the relative sizes of the prey and predator, varying from about fifteen minutes to about two hours. For all stages with a length greater than $4 \mathrm{~mm}$, the buccal apparatus is effective on a large size range of prey. The intensity of predation by larvae of $A$. ibis on a population of older Chironomidae is a linear function of the size of the predators between 5 and $15 \mathrm{~mm}$.

Cannibalism rarely occurs when the larvae are not deprived of food. The older specimens can endure a period of six months without food at $10-15^{\circ} \mathrm{C}$.

Some observations have also been made on the food of Atrichops crassipes.

1. Laboratoire d'Hydrobiologie, Université Paul-Sabatier, 118, route de Narbonne, 31077 Toulouse Cedex, France. 


\section{I. - INTRODUCTION ${ }^{1}$. CARACTẼRES GÉNÊRAUX}

Le régime alimentaire des Athericidae n'est connu que grâce à quelques observations sommaires de Nagatomi (1962).

L'appareil buccal, de type perforant-suceur, et le mode de nutrition sont voisins de ceux des Tabanidae. Les deux caractè-

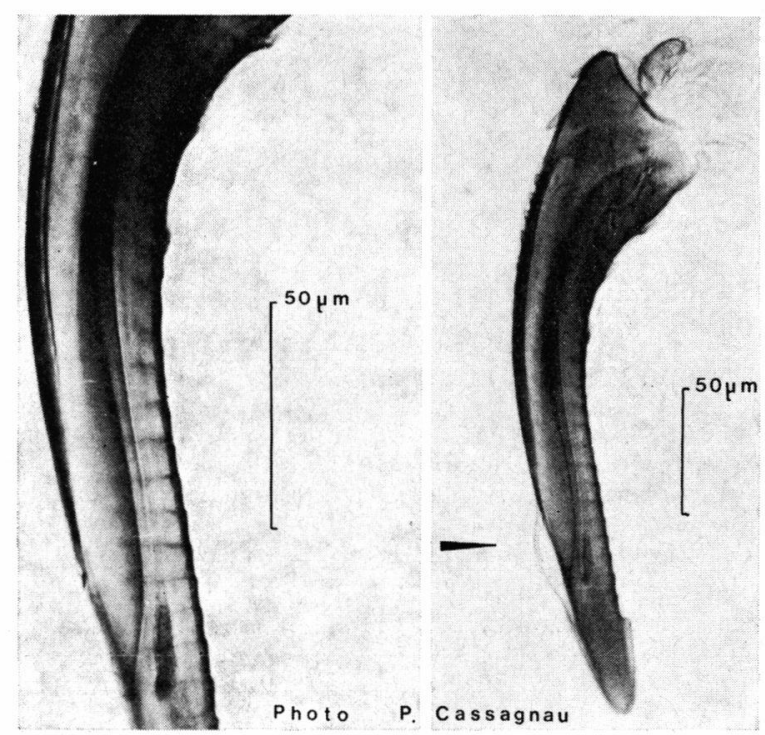

Pното 1. - Mandibule canaliculée d'une larve d'A. ibis de $22 \mathrm{~mm}$.

res principaux des trois espèces sont la présence de mandibules pivotantes perforantes, canaliculées [photo 1 : comparer à Wyniger (1953, p. 341) et à Teskey (1969, p. 12)] et l'obligation d'ingérer une nourriture fluide. Le tube digestif ne renferme donc aucun débris chitineux qui permettrait d'étudier le régime alimentaire.

La perforation des téguments de la proie est aussitôt suivie d'une injection très toxique (Schremmer 1951). Il est aisé d'observer qu'un Chironomidae de même longueur que l'Athericidae est presque toujours paralysé ou tué en moins d'une seconde. Une digestion partielle extraorale liquéfie ensuite les tissus internes de la proie, excepté en général le tube digestif et son contenu. Cette liquéfaction peut être facilement constatée en séparant le prédateur de sa proie. Dans un dernier temps, l'Athericidae vide celle-ci par succion. Liquéfaction et succion se font plus ou

1. Position systématique et description des trois espèces du Sud de la France : Thomas, 1974 a et b. 
moins complètement selon la nature de la proie et la taille relative des deux animaux en présence.

Ces observations sont donc assez voisines de celles faites par plusieurs auteurs sur la nutrition des larves d'autres Brachycères Orthorrhaphes, notamment par Cameron (1934) sur Haematopota pluvialis (Tabanidae) el Tsacas (1962) sur Chrysopilus auratus (Rhagionidae).

Wyniger (1953) a montré l'action protéolytique de la salive des larves de Tabanidae, en particulier sur les érythrocytes, les lymphocytes, les cellules chloragogènes et les fibres musculaires.

Les Athericidae diffèrent aussi des autres invertébrés torrenticoles prédateurs par leur comportement. Les Atherix s.s. en particulier vivent surtout sous les pierres (Hynes 1970). Observés dans un ruisseau artificiel à fond transparent, ils pénètrent profondément, avec obstination, dans les interstices entre les cailloux ou les graviers. Les Athericidae ne possèdent d'autre part pas d'éléments chitineux volumineux comparables par exemple à la capsule céphalique, au tergite prothoracique et aux pattes articulées des Trichoptères carnivores du genre Rhyacophila (largeurs des capsules céphaliques de specimens de $20 \mathrm{~mm}$ : Rhyacophila evoluta $=1,6 \mathrm{~mm}, A$. ibis $=0,3 \mathrm{~mm}$ ). Leur tégument, quoique résistant, est d'une très grande souplesse, nettement supérieure à celle des téguments des Tabanidae et des Rhagionidae du genre Chrysopilus rencontrés dans les cours d'eau. Ils peuvent ainsi atteindre, beaucoup plus facilement que la plupart des autres prédateurs de même taille, des proies de petites dimensions ou de faible section transversale, adaptées précisément aux interstices (Plécoptères Leuctridae par exemple). J'ai pu d'ailleurs vérifier que l'appareil buccal des larves reste, jusqu'au dernier stade, efficace sur des proies de faible diamètre.

Les concurrents les plus directs des Athericidae devraient donc être, à priori, surtout les Diptères Limoniidac des genres Hexatoma et Dicranota - ces derniers cependant de taille notablement inférieure — dans les fonds pierreux et du genre Eloeophila dans les zones ensablées.

\section{II. - METTHODE EMPLOYẼE}

Seul sera étudié ici l'aspect qualitatif de la prédation chez les larves d'Athericidae. Quelques 150 expériences ont été effectuées, la plupart pendant la nuit, sur plusieurs milliers d'invertébrés torrenticoles divers et plus de 600 larves d'Athericidae de taille comprise entre 3 et $30 \mathrm{~mm}$. La plus grande partie du travail a été conduite sur Atherix ibis (environ 500 larves), l'espèce la plus grande et la plus aisée à observer. Quelques expériences complémentaires suggèrent que le comportement prédateur d'A. 
marginata et même celui d'Atrichops crassipes - espèce nettement psammophile et de taille bien plus petite - ne présentent pas de différences fondamentales avec celui d'A. ibis.

Ces observations ont été faites sur fonds de pierres ou de graviers (biotopes les plus riches en $A$. ibis dans les Pyrénées centrales). La prédation sur quelques formes franchement muscicoles a été étudiée dans des touffes de Bryophytes aquatiques immergées. Deux séries d'expériences ont été effectuées :

1) en eau courante, dans des gouttières d'écoulement à fond plat transparent ou à fond opaque en $\mathrm{V}$ ou en $\mathrm{U}$ plus ou moins accentués (Thomas 1975). Pente, débit el granulométrie du fond - conditionnant la taille des abris - ont pu être adaptés, dans une certaine mesure, aux invertébrés étudiés.

Pente : $<1$ à $15 \%$; débil : 0,1 à $6 \mathrm{l} / \mathrm{mn}$; vitesse moyenne en surface : 0 à $50 \mathrm{~cm} / \mathrm{s}$; granulométric : 5 à $150 \mathrm{~mm}$.

Analyse de l'eau, effectuée le 15-9-1975 (mg/l) :

$$
\begin{array}{ccccccccc}
\mathrm{HCO}_{3}{ }^{-} & \mathrm{Ca}++ & \mathrm{NO}_{3}-\mathrm{N} & \mathrm{SiO}_{2} & \mathrm{PO}_{4}-\mathrm{P} & \mathrm{K}+ & \mathrm{Mg}++ & \mathrm{Na}+ \\
196 & 64 & 0,31 & 5,7 & 0,001 & 2 & 5,5 & 5,5
\end{array}
$$

Résistivité à $20{ }^{\circ} \mathrm{C}: 2516 \Omega / \mathrm{cm}^{2} / \mathrm{cm}$.

La température de l'eau a varié au cours des expériences de 10 à $19^{\circ} \mathrm{C}$.

2) en eau non renouvelée, mais fortement oxygénée par des pompes à air, dans des cuvettes en matière plastique plus ou moins inclinées; abris constitués par des cailloux, graviers ou morceaux de bois flotté de taille comprise entre 20 et $100 \mathrm{~mm}$.

Analyse de l'eau utilisée, effectuée le 15-9-1975:

$$
\begin{array}{cccccccc}
\mathrm{HCO}_{3}{ }^{-} & \mathrm{Ca}^{+}+ & \mathrm{NO}_{3}-\mathrm{N}^{-} & \mathrm{SiO}_{2} & \mathrm{PO}_{4}-\mathrm{P} & \mathrm{K}+ & \mathrm{Mg}^{++} & \mathrm{Na}^{+} \\
411 & 134 & 0,62 & 5,1 & 0,002 & 2,2 & 42 & 2,25
\end{array}
$$

Résistivité à $20^{\circ} \mathrm{C}: 1099 \Omega / \mathrm{cm}^{2} / \mathrm{cm}$.

La température de l'eau a varié, de mai à septembre, de 16 à $23^{\circ} \mathrm{C}$.

Des boîtcs de Pétri avec une faible hauteur d'enu, inférieure à $5 \mathrm{~mm}$, ont aussi été utilisécs à température ambiante pour l'observation directe de l'attaque et de la succion, en particulier chez Atrichops crassipes, psammophile fouisseur qui n'aurait guère pu être étudié dans le sable. Le support des animaux a alors été réalisé par des fragments de mousse de plastique.

Les longueurs citées s'entendent sans les antennes (Gammares, Plécoptères), sans les cerques (Plécoptères, Ephéméroptères) et sans les prolongements postérieurs (A. crassipes).

Je remercie pour leurs déterminations MM. C. Bertélemy (Plécoptères, Coléoptères), H. Décamps (Trichoptères), N. Giani (Oligochètes) et $\mathrm{H}$. Laville (Diptères Chironomidae). 


\section{III. - RELATIONS TROPHIQUES \\ AVEC LES DIVERS GROUPES D'INVERTÉBRÉS AQUATIQUES DES MEMES BIOTOPES}

\section{- Planaires, Hirudinées et Limnées.}

Aucune expérience concluante. Les Athericidae n'attaquent pas, semble-t-il, les Invertébrés qui sécrètent de grandes quantités de mucus.

\section{- Oligochètes.}

Les Oligochètes constituent une part probablement importante du régime alimentaire de beaucoup de Tabanidae et de Rhagionidac : ils sont fréquemment utilisés lors de l'élevage des larves de ces Diptìres (Marchand 1917', Cameron 1934, Schwardt 1937, Wyniger 1953, Chvála et coll. 1972, F. Vaillant comm. verb.). Mais en dépit de nombreux élevages, parfois de longue durée, les $A$. ibis et $A$. crassipes privés de nourriture n'ont jamais sucé ni même tué d'Oligochètes. Ceux-ci² ont été volontairencnt choisis de taille très variable $(10$ à $50 \mathrm{~mm})$. A chaque fois, l'Athericidae paraît éviter au dernier moment le contact de l'Annélide, ce qui est peut être en rapport avec le mucus de ce dernier. Or, le remplacement des Oligochètes par des Chironomides a toujours entraîné une attaque immédiate de la part des Atrichops. Cette espèce ne paraît donc pas consommer les nombreux Oligochètes de petite taille avec lesquels elle vit pendant la plus grande partie de son cycle de développement.

\section{- Amphipodes.}

Les Gammares ne constituent que rarement une proie pour les A. ibis el peuvent cohabiter avec ceux-ci pendant longtemps. Le tégument de ces Amphipodes, épais et glissant, ne se prête pas à la perforation sur les faces dorsale et latérales. Taille des specimens utilisés: Gammares de 3 à $17 \mathrm{~mm}, A$. ibis de 18 à $28 \mathrm{~mm}$. Quant aux perforations ventrales, elles sont possibles mais vraisemblablement très rares. Moins exceptionnelles sont les attaques frontales qui peuvent déplacer le céphalon du crustacé el permettre une perforation sur la membrane articulaire,

1. Cité par Isaac (1924).

2. Espèces utilisées : avec A. ibis, Eiseniella tetraedra variété hercynia (Lumbricidae) et Limnodrilus udekemanius (Tubificidae); avec A. crassipes : Tubifex ignotus, T. tubifex, Limnodrilus sp. (Tubificidae). 
à la base du premier péréionite. Ce dernier cas paraît être la seule possibilité pratique pour un Athericidae de venir à bout d'un Gammare de plus de 9 ou $10 \mathrm{~mm}$. Observations effectuées en eau courante $\left(12\right.$ à $\left.14^{\circ} \mathrm{C}\right)$ et en eau non renouvelée $\left(18\right.$ à $\left.21^{\circ} \mathrm{C}\right)$.

\section{- Plécoptères.}

Les Plécoptères n'interviennent qu'assez secondairement dans l'alimentation des Athericidae. Quelques observations ont été conduites en eau courante $\left(12\right.$ à $\left.16{ }^{\circ} \mathrm{C}\right)$ et en eau non renouvelée $\left(19-20^{\circ} \mathrm{C}\right)$ sur les familles des Taeniopterygidae, Nemouridae, Leuctridae, Perlodidae, Perlidae et Chloroperlidae avec des A. ibis de 11 à $18 \mathrm{~mm}$.

Lorsque ces prédateurs n'ont subi qu'un jeûne court (moins d'une semaine) et qu'ils ont à leur disposition une grande variété de proies, les cas de succion de Plécoptères d'une longueur supérieure à 6 ou $7 \mathrm{~mm}$ sont assez peu fréquents. Il n'en n'est pas de même si les $A$. ibis sont à jeun depuis plus longtemps (2 semaines à $20^{\circ} \mathrm{C}$ ) et s'il ne leur est pas offert d'autres proies. Dans ce cas, les trois familles Nemouridae, Leuctridae et Chloroperlidac présentent la mortalité la plus élevée (exemple : larves âgées de Protonemura beatensis de 6 à $8 \mathrm{~mm}$, Leuctra geniculata et Leuctra $s p$. de 6 à $8 \mathrm{~mm}$, de Siphonoperla torrentium et Chloroperla $s p$. de 7 à $9 \mathrm{~mm}$ ).

Les Perlodidae ne sont tués et sucés qu'après un jeûne prolongé du Diptère et leur taille est en général inférieure à celle de l'Atherix (exemple : Isoperla acicularis de 11 à $13 \mathrm{~mm}$, l. sp. dı groupe grammatica de 9 à $11 \mathrm{~mm}$ ). Les Perlidae ne sont vulnérables que lors des stades jeunes : des cas de prédation ont été notés sur des larves de Perla marginata jusqu'à $12 \mathrm{~mm}$. Dinocras cephalotes, à taille égale et à plus forte raison supérieure, paraît à peu près invulnérable face aux Athericidae. Les larves de cette espèce, très vives, peuvent pour une longueur de 15 à $25 \mathrm{~mm}$, cohabiter plusieurs semaines en eau courante avec des $A$. ibis de 25 à $30 \mathrm{~mm}$ maintenus à jeun. Un cas de prédation a été observé sur un specimen de $7 \mathrm{~mm}$, au dernier stade, de l'espèce assez rare Taeniopteryx nebulosa (Taeniopterygidae).

Le tégument des Plécoptères est presque toujours perforé sur les faces latérales ou inférieure du thorax, parfois aussi entre tête et prothorax. On note également, à la suite d'attaques par l'arrière, des pénétrations entre les sclérites de l'abdomen, sur les membranes articulaires.

\section{- Ephéméroptères.}

Sur fond pierreux, ce sont probablement les insectes qui jouent le rôle le plus important dans la nutrition des Atherix. Ceci peut 
être mis en évidence en plaçant avec des $A$. ibis plusieurs specimens des différents ordres de la même station. Néanmoins, la prédation exercée sur les différents genres d'Ephéméroptères est, à taille égale, très variable. Il semble que l'épaisseur de leur cuticule soit déterminante. Les représentants des Baetidae, des Heptageniidae et, dans une moindre mesure, des Leptophlebiidae sont de loin les plus touchés dans les élevages lorsque les larves d'A. ibis ont la possibilité de choisir leurs proies. Au contraire, les Ephemerellidae, fréquents dans les ruisseaux et rivières de basse altitude dans les mêmes milieux que ce prédateur, ne sont, malgré leurs mouvements lents, que beaucoup plus rarement lués - du moins au-delà d'une longueur de l'ordre de $6 \mathrm{~mm}$. Les Ephemera de plus de $10 \mathrm{~mm}$ sont peu vulnérables vis-à-vis des $A$. crassipes.

Les zones de perforation préférentielles sont les mêmes que chez les Plécoptères mais la digestion extraorale est en général plus complète surtout dans la partie terminale de l'abdomen, sauf pour les genres Ephemerella et Torleya.

Exemple 1: $18 \mathrm{~A}$. ibis de 19 à $26 \mathrm{~mm}$, capturés le jour même, ont vidé du 16-5-1974, $22 \mathrm{~h}$ au 17-5, $22 \mathrm{~h}: 8$ Baetis alpinus de 7 à $10 \mathrm{~mm}$, 13 Rhithrogena sp. de 7 à $9 \mathrm{~mm}, 3$ Epeorus torrentium de 8 à $9 \mathrm{~mm}$, 3 Ecdyonurus sp. de 9 à $13 \mathrm{~mm}$, 2 Habroleptoides berthelemyi de $8 \mathrm{~mm}$. Eau courante, $14^{\circ} \mathrm{C}$.

Exemple $2: 6$ A. ibis (1 de $18 \mathrm{~mm}, 3$ de $23 \mathrm{~mm}, 1$ de $25 \mathrm{~mm}, 1$ de $28 \mathrm{~mm}$ ), prélevés le jour même, ont sucé du 18-4-1975, $19 \mathrm{~h}$ au 19-4, $9 \mathrm{~h}: 4$ Baetis rhodani de 6 à $8 \mathrm{~mm}, 9$ Rhithrogena $s p$. de 10 à $13 \mathrm{~mm}$, 5 Epeorus torrentium de 15 à $17 \mathrm{~mm}$. En revanche, les 3 Ephemerella ignita de 6 à $8 \mathrm{~mm}$ ajoutés également ont survécu. Eau courante, $13^{\circ} \mathrm{C}$.

Exemple 3:1 Ephemerella ignita le $7 \mathrm{~mm}$, au dernier stade, mis avec $70 \mathrm{~A}$. ibis de taille comprise entre 11 et $18 \mathrm{~mm}$ à jeun depuis une semaine, parvient à l'émergence au bout de 5 jours. Eau stagnante, $20^{\circ} \mathrm{C}$, surface du fond de $250 \mathrm{~cm}^{2}$ seulement.

\section{- Trichoptères.}

Face aux Atherix, les Trichoptères torrenticoles du sud de la France peuvent être classés schématiquement dans les catégories suivantes :

- formes à fourreau;

- formes libres, à filet;

- formes libres, sans filet.

1) Espèces à fourreau.

La protection contre les Atherix est efficace, la capsule céphalique obturant l'ouverture du fourreau lors du retrait du corps dans celui-ci. La perforation n'est possible que sur des individus en déplacement, dont le thorax n'est donc plus protégé : le plus 
souvent elle est faite immédiatement en arrière du prothorax (exemples: quelques larves de Sericostomatidae de 10 à $16 \mathrm{~mm}$ et de Potamophylax sp. (Limnephilidae) de 12 à $18 \mathrm{~mm}$ ). Mais en général les espèces à fourreau peuvent cohabiter longtemps (plus d'un mois) avec des $A$. ibis privés de nourriture (exemple : larves d'Halesus sp. et Potamophylax sp. de 13 à $19 \mathrm{~mm}$ ). Eau courante, températures comprises entre 12 et $15^{\circ} \mathrm{C}, A$. ibis de 14 à $26 \mathrm{~mm}$.

Une tentative de perforation par l'orifice de la base du fourreau a été notée (Potamophylax sp.).

2) Espèces libres à filet.

Expériences menées sur les trois familles Philopotamidae, Hydropsychidae et Polycentropodidae. Deux possibilités ont été examinées :

- Ies Trichoptères sont placés dans un habitat déjà colonisé par les Atherix, ce qui peut reproduire dans une certaine mesure le cas d'animaux en dérive ;

- les Trichoptères maintenus au préalable pendant 12 heures à une température inféricure de 6 à $7^{\circ} \mathrm{C}$ à la température de l'expérience proprement dite, colonisent un habitat vierge. Ils y sont laissés $36 \mathrm{~h}$ avec une vitesse de courant faible, de l'ordre de $5 \mathrm{~cm} / \mathrm{s}$. Ces conditions sont favorables à l'établissement de filets (H. Décamps, comm. verb., Tachet 1975). Les Atherix ne sont ajoutés qu'après.

a) Philopotamidae.

Malgré leurs puissantes mandibules et leur vivacité, ils sont - avec ou sans filets - - très vulnérables et entrent sans doute pour une part importante dans l'alimentation d'A. ibis (nombreuses observations).

Exemple 1 : le 15-11-1973 à 18 h 30, 25 Chimarra marginata de 11 à $13 \mathrm{~mm}$ sont mis avec 25 A. ibis de 12 à $16 \mathrm{~mm}$ à jeun depuis 10 jours. 16-11, $9 \mathrm{~h}: 21$ Chimarra sucés ; 29 autres sont alors ajoutés. 17-11, $9 \mathrm{~h}$ : 9 Chimarra sucés. 18-11, $9 \mathrm{~h}: 10$ Chimarra sucés soit un total de 40. Aucun A. ibis n'a été tué. Température : $14^{\circ} \mathrm{C}$. Eau courante.

Exemple 2 : le $1-9-1975$ à $18 \mathrm{~h}$ sont placés en eau courante à $17^{\circ} \mathrm{C}$ 13 Chimarra marginata de 10 à $15 \mathrm{~mm}$ et 1 Philopotamus sp. de $14 \mathrm{~mm}$, maintenus depuis $12 \mathrm{~h}$ à $10^{\circ} \mathrm{C}$. 40 heures après sont ajoutés $14 \mathrm{~A}$. ibis de 10 à $21 \mathrm{~mm}$ provenant du même prélèvement (donc sans jeûne expérimental). Le 4-9 à $18 \mathrm{~h}$, il ne reste que 2 Chimarra vivants. La présence d'un filet auprès de chaque cadavre est constatée. Aucun Alherix n'a été tué.

\section{b) Hydropsychidac.}

Leur tégument abdominal, plus épais que celui des Philopotamidae, est moins fréquemment perforé, sauf dans la zone latéro- 
ventrale, un peu en arrière de la $3^{\circ}$ paire de pattes. Plusieurs cas de prédation ont été observés sur des spécimens de 12 à $17 \mathrm{~mm}$ dépourvus de filets ( $A$. ibis de $16 \mathrm{~mm}, 13^{\circ} \mathrm{C}$ ).

Les spécimens abrités par leurs filets sont susceptibles de cohabiter avec des $A$. ibis nourris. Maintenus à jeun, ces derniers poursuivent les Hydropsyche - malgré les morsures de ceux-ci jusque dans leurs filets que les Trichoptères finissent par abandonner à reculons, en faisant face constamment. Les Atherix se débarrassent parfois très difficilement du filet vide.

\section{c) Polycentropodidae.}

Observations faites sur des larves de Plectrocnemia sp. Ce genre est prédateur et son opposition à $A$. ibis est intéressante bien que les deux formes cohabitent peu dans les cours d'eau.

Sans filet, ces larves se révèlent étonnament vulnérables malgré leur très grande vivacité, sans doute à cause de la minceur de leur tégument abdominal.

Exemple : 6 Plectrocnemia $s p$. de 16 à $20 \mathrm{~mm}$ sont mis avec $7 \mathrm{~A}$. ibis de 17 à $21 \mathrm{~mm}$ le $4-12-1973$ à $10 \mathrm{~h}$. Le $5-12$ à $9 \mathrm{~h}, 5$ ont été sucés. Aucun Atherix n'a été tué. Eau courante, $13^{\circ} \mathrm{C}$.

Abrités par leurs filets, les Plectrocnemia se montrent au contraire d'une telle agressivité que les $A$. ibis en chasse à l'entrée du filet finissent par reculer. Il arrive souvent que les nombreux crochets de leurs pseudopodes s'emmêlent dans les fibres, l'Atherix devenant pendant quelques instants une proie à son tour. Les morsures des Plectrocnemia, pour des tailles relatives du même ordre, paraissent cependant sans effet sur le tégument souple du Diptère. Ainsi, un Plectrocnemia $s p$. de $19 \mathrm{~mm}$ a mordu à plus de trente reprises en 10 minutes un $A$. ibis de $21 \mathrm{~mm}$ sans lui causer la moindre blessure visible à un grossissement de 80 diamètres.

\section{3) Espèces libres sans filet.}

Le genre le plus répandu est Rhyacophila, prédateur.

D'une façon générale, les Rhyacophila spp. et les $A$. ibis s'évitent dans les élevages quand ils disposent de proies en nombre suffisant. Lorsque par suite du jeûne, l'affrontement se produit, A. ibis l'emporte le plus souvent à taille égale ou légèrement inférieure. Dans des conditions naturelles et pour un relief donné, grâce à sa plus grande souplesse et aux dimensions réduites de ses pièces rigides, l'Atherix a sans doute souvent soit l'avantage de la taille sur beaucoup d'espèces de Rhyacophila soit la possibilité de se mettre à l'abri. 
La plupart des observations ont été effectuées sur la plus grande espèce locale : Rh. martynovi.

Exemple 1 : entre le 14-12-1973, $23 \mathrm{~h}$ et le 15-12, $9 \mathrm{~h}, 1 \mathrm{~A}$. ibis de $19 \mathrm{~mm}$ tue et suce partiellement $1 \mathrm{Rh}$. martynovi de $29 \mathrm{~mm}$.

Exemple $2: 1$ A. ibis de $25 \mathrm{~mm}$, à jeun depuis 8 semaines, suce partiellement du 19-12-1973, $9 \mathrm{~h}$. au 20-12, $9 \mathrm{~h}, 1 \mathrm{Rh}$. dorsalis de $20 \mathrm{~mm}$, puis du $27-12,16 \mathrm{~h}$ au $28-12,9 \mathrm{~h}, 1 \mathrm{Rh}$. martynovi de $28 \mathrm{~mm}$, enfin du 9-1-1974, $11 \mathrm{~h}$ au 10-1, $15 \mathrm{~h}, 1 \mathrm{Rh}$. martynovi de $25 \mathrm{~mm}$. Aucune nourriture n'a été fournie à l'Atherix dans l'intervalle.

Dans les deux cas : eau courante, $12{ }^{\circ} \mathrm{C}$.

\section{— Diptères.}

La plupart des larves de Brachycères torrenticoles vivent dans les mousses, le sable ou les rives dans la zone de battement de l'eau. Leur tégument est le plus souvent très épais. Seules seront donc considérées ici les larves de Nématocères, plus nettement rhéophiles et adaptées aux fonds pierreux.

\section{1) Simuliidae et Blepharoceridae.}

Les Simulies sont très vulnérables.

Exemple : Ie 22-4-1973 un lot de Simulies de 3,5 à $5 \mathrm{~mm}$ est placé avec $21 \mathrm{~A}$. ibis de 22 à $27 \mathrm{~mm}$. Le $23-4,9 \mathrm{~h}, 221$ ont été sucées, presque toutes complètement, 18 seulement survivent. Eau courante, température $14{ }^{\circ} \mathrm{C}$.

En élevage, les cas de prédation sur les Blepharoceridae sont assez fréquents. Mais, plus encore que pour les Simuliidac, ils sont probablement peu significatifs. A la différence des Atherix, les Blepharoceridae vivent, en effet, surtout à la face supérieure des pierres et sur les roches en place en courants rapides à violents.

\section{2) Chironomidae.}

Les Chironomidae constituent une proie particulièrement facile pour les trois espèces d'Athericidae, même au dernier stade (tableau 1).

\section{3) Limoniidae.}

A. ibis se nourrit éventuellement de Dicranota spp. et d'Hexatoma bicolor, prédateurs plus petits que lui.

A. crassipes attaque parfois, mais semble perforer avec difficulté les Eloeophila spp., prédateurs psammophiles, qui vivent avec lui.

Il a été noté à deux reprises unc grande résistance vis-à-vis de l'injection paralysante : 
Exemple 1: 1 Hexatoma bicolor de $16 \mathrm{~mm}$, attaqué par un A. ibis de $24 \mathrm{~mm}$, bouge encore $30 \mathrm{mn}$ après la perforation et est pratiquement sucé vivant (il se contracte au contact des pinces au bout d'une heure, sa longueur déjà réduite d'un tiers). Eau courante, $13^{\circ} \mathrm{C}$.

Exemple 2: 1 Eloeophila sp. de $13 \mathrm{~mm}$, perforé par un A. crassipes de $9 \mathrm{~mm}$, se débat par intermittence pendant $10 \mathrm{mn}$, finit par se libérer et s'échapper. Eau stagnante, $18^{\circ} \mathrm{C}$.

4) Tipulidae s. s.

Les espèces pyrénéennes torrenticoles rencontrées dans les mêmes milieux que les $A$. ibis appartiennent au groupe Tipula saginata, fréquemment muscicole.

Les perforations sont rares sur le stade IV qui atteint $35 \mathrm{~mm}$ (l'Atherix dépasse en général $25 \mathrm{~mm}$ ). Fait assez surprenant, clles sont peu fréquentes aussi sur les stades II et III malgré des élevages de plus de deux semaines. Les expériences ont été surtout conduites dans des Bryophytes aquatiques avec une faible hauteur d'eau : les Atherix avaient constamment accès aux Tipulidae dont la cupule respiratoire reste en surface. Eau courante à $13^{\circ} \mathrm{C}$, eau stagnante, 18 à $21{ }^{\circ} \mathrm{C}$.

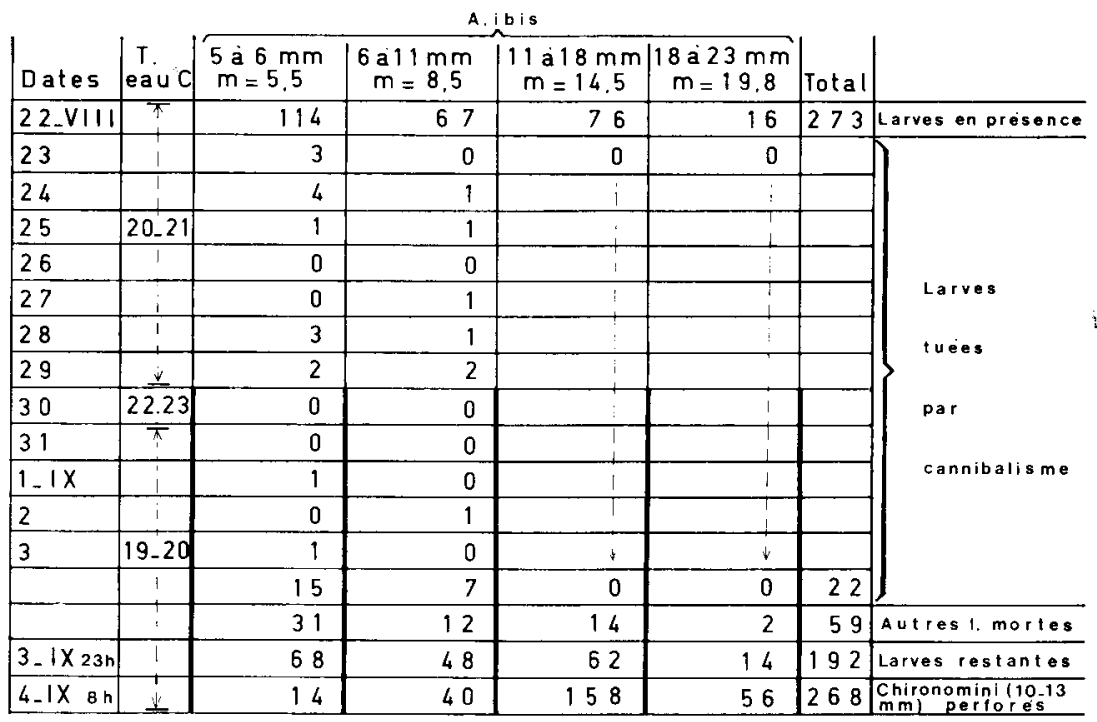

Tableau 1. - Cannibalisme chez une population non nourrie de 273 A. ibis en 12 jours. Impact des survivants sur une population de Chironomus thummi thummi en une nuit. Les traits épaissis correspondent à la séparation des larves d'A. ibis en classes de taille.

\section{- Coléoptères.}

Les larves et adultes de la plupart des Coléoptères d'eau courante ne sont pas consommées par les Atherix.

Les expériences ont porté sur des adultes d'Hydraena gracilis (Hydraenidae), Elmis maugetii, Riolus cupreus, Limnius opacus 
et L. volkmari (Elminthidae) et sur des larves âgées de ces deux dernières espèces. Aucune perforation n'a pu être observéc avec des A. ibis de 6 à $26 \mathrm{~mm}$.

Par contre, les larves d'Hydrociphon (Hclodidae), moins sclérifiées, sont parfois vulnérables, comme les Gammares, aux attaques frontales qui permettent une perforation entre tête et pronotum. Eau courante à $15^{\circ} \mathrm{C}$, eau stagnante, 19 à $22^{\circ} \mathrm{C}$.

\section{- Insectes prédateurs d'Atherix ibis.}

Ils sont peu nombreux dans les eaux courantes du Sud-Ouest de la France. Ne seront considérés ici que les prédateurs de larves de plus de $15 \mathrm{~mm}$, diverses espèces omnivores pouvant probablement consommer les jeunes stades d'Atherix. Il ne sera pas tenu compte non plus des prédateurs possibles dans des milieux où l'Atherix n'est rencontré qu'accidentellement (Nepidae, Tabanidae psammophiles, etc.).

\section{1) Plécoptères.}

Aucun cas de prédation par les Subulipalpia n'a pu être observé pendant six expériences effectuées en eau courante (température 11 à $13^{\circ} \mathrm{C}$ ) sur une durée atteignant 10 jours. La face inférieure des pierres utilisées était concave et permettait le passage et l'abri de larves âgées de trois espèces de grande taille, maintenues à à jeun : Perla grandis jusqu'à $30 \mathrm{~mm}, P$. marginata et Dinocras cephalotes. Mais l'élevage des deux espèces de Perla s'est révélé assez délicat, les larves paraissant peu actives, tout au moins dans nos conditions d'éclairement. Taille des A. ibis : 15 à $19 \mathrm{~mm}$.

D'autre part, aucun débris d'Athericidac ne figure dans les contenus stomacaux de plusieurs dizaines de Perla provenant de la même région (C. Berthélemy, comm. verb.).

2) Trichoptères.

Le genre Rhyacophila est le prédateur d'A. ibis le plus fréquent lorsque la différence de taille est nettement en faveur du Trichoptère.

Exemple 1 : du 15-12-1973, 23 h au 16-12, 9 h, 1 Rh. martynovi de $32 \mathrm{~mm}$ a éventré n plusieurs endroits un $A$. ibis de $19 \mathrm{~mm}$.

Exemple 2 : du 15-12-1973, 9 h au 17-2, $9 \mathrm{~h}, 5 \mathrm{Rh}$. martynovi de 30 à $35 \mathrm{~mm}$ mis avec 13 A. ibis de 17 à $21 \mathrm{~mm}$ en ont tué 3 . Les 10 survivants se sont déplacés tout à fait en aval de la gouttière d'écoulement.

Dans les deux cas : eau courante, $12{ }^{\circ} \mathrm{C}$.

3) Diptères.

Les larves de Chrysopilus paraissent être les seuls prédateurs fréquents d'Atherix, malgré la lenteur de leurs mouvements. 
Exemple : le 18-4-1975 entre 11 et $12 \mathrm{~h}, 1$ Chrysopilus sp. de $25 \mathrm{~mm}$ a sucé $1 \mathrm{~A}$. ibis de $17 \mathrm{~mm}$. Milieu : Bryophytes aquatiques en eau stagnante à $16^{\circ} \mathrm{C}$.

Dans ce milieu, les A. ibis sont certainement aussi la proie des Muscides muscicoles du genre Limnophora.

\section{IV. - LE CANNIBALISME}

Rare, le cannibalisme ne se rencontre dans les élevages que dans des conditions de surpopulation et de nutrition très défavorables. En eau courante - et même en eau non renouvelée — des spécimens de tailles nettement différentes peuvent cohabiter pendant plusieurs semaines sans nourriture. Les larves limitent alors beaucoup leurs déplacements, se recroquevillent côte à côte sous les pierres (photo 2) et attendent des conditions plus favorables. Il a ainsi pu être dénombré, en cas de carence en abris, jusqu'à 47 larves d'A. ibis de 11 à $18 \mathrm{~mm}$ sous une pierre de $4,20 \mathrm{~cm}^{2}$ seulement, soit 11 larves au $\mathrm{cm}^{2}$, contiguës et réparties en trois ou quatre épaisseurs pendant plusieurs jours sans cannibalisme! Même dans de meilleures conditions, le comportement des larves d'Atherix, à quelque stade que ce soit, ne met en évidence aucun instinct territorial net et demeure très différent de celui de beaucoup d'insectes prédateurs, des Rhyacophila en particulier.

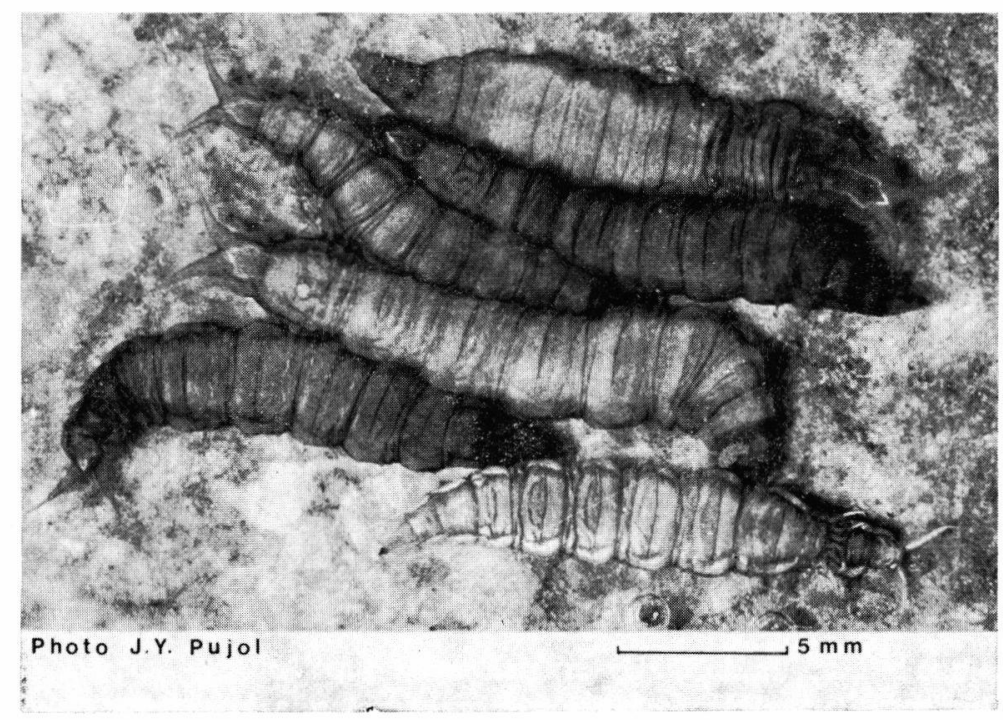

Рното 2. - Attitude collective de larves d'A. ibis au repos sous une pierre. Vue prise par dessous, dans un ruisseau expérimental à fond transparent. 
Exemple 1: 12 A. ibis de longueur comprise entre 14 et $22 \mathrm{~mm}$, placés en eau courante, sans nourriture, de janvier à mai, n'ont sucé qu'un seul d'entre eux au terme de ces 17 semaines. Une expérience semblable a été faite au même moment et dans les mêmes conclitions avec 7 larves d'A. marginata, de longueur comprise entre 9 et $13 \mathrm{~mm}$. Toutes ont survécu. La température de l'eau a varié de 12 à $16^{\circ} \mathrm{C}$. Surface du fond : $250 \mathrm{~cm}^{2}$.

Exemple $2: 2 \mathrm{~A}$. ibis de 15 et $21 \mathrm{~mm}$ laissés à $10^{\circ} \mathrm{C}$ à l'obscurité dans un flacon ouvert (surface de base $25 \mathrm{~cm}^{2}$ ), avec peu d'eau non renouvelée et sans nourriture, pendant 13 semaines (de mai à août) sont retrouvés vivants et actifs.

Exemple 3: le 7-6-1975, 62 A. ibis de taille comprise entre 8 et $22 \mathrm{~mm}$, à jeun depuis au moins 28 jours (prélèvement du 10-5) ont été mis en eau stagnante sur une surface de $250 \mathrm{~cm}^{2}$ seulement. Le 15-7 il n'en reste que 29 en vie. Aucune proie n'a été donnée. La température de l'eau à été comprise entre 20 et $23^{\circ} \mathrm{C}$ et la plupart des cadavres - retirés chaque jour - sont plus ou moins nettement couverts de mycéliums. 5 sur 33 seulement portent des traces de perforations, alors que les survivants sont presque tous très actifs.

Exemple 4 : le tableau 1 montre qu'en cas de surpopulation intense - 0,5 larve au $\mathrm{cm}^{2}$ — par température élevée et sans nourriture, le cannibalisme est incontestable mais s'exerce aux dépens des stades jeunes : il est fortement atténué lorsque les animaux sont répartis en classes de taille (traits épaissis). Dans les mêmes conditions, ces Atherix ont tué 268 Chironomus thummi thummi en une nuit. Cette hécatombe montre bien, par comparaison, le peu d'importance du cannibalisme. Ceci confirme l'hypothèse formulée par Nagatomi (1962).

Le fait de provoquer une blessure sur une larve (section d'une fausse patte ou d'un prolongement caudal, boutonnière sur le tégument) ne paraît pas rendre celle-ci plus vulnérable vis-à-vis de ses congénères.

En définitive, si le cannibalisme existe - il a été signalé également chez A. marginata (Van̆hara 1975) - dans des conditions de nutrition et de densité de population précaires et artificielles, il paraît en pratique fort douteux dans les cours d'eau. Son rôle sur les biomasses d'Atherix est probablement à peu près nul. La notion de cannibalisme est incontestable chez certains Diptères Brachycères, en particulier les Tabanidae (Surcouf 1924, Vaillant 1955, Chvála et coll. 1972, etc.) ; elle ne saurait cependant être étendue à tous les genres d'Orthorrhaphes possédant des espèces aquatiques torrenticoles. Les présentes observations vont au contraire dans le sens de celles de Tsacas (1962) sur Chrysopilus auratus (Rhagionidae) et de celles de Chvála et coll. (1972) sur le genre Chrysops (Tabanidae).

\section{V. - DISCUSSION}

\section{1) Tailles limites de la proie.}

Elles ont été étudiées sur des Éphéméroptères et des Chironomidae. Les valeurs extrêmes ont été observées plusieurs fois. 
- Taille maximale de la proie : les A. ibis âgés sont capables de tuer des proies de leur taille ou plus grandes qu'eux (Rhyacophila). De même, les larves jeunes, à $6 \mathrm{~mm}$, tuent des Baetis et des Rhithrogena de $7 \mathrm{~mm}$ ou des Chironomini de $13 \mathrm{~mm}$. Dans ce cas, ils n'absorbent pas tout le contenu de leur proic sauf si deux ou plusieurs Atherix vident le même individu.

- Taille minimale de la proie : un A. ibis de $20 \mathrm{~mm}$ peut sucer des Baetis de $2 \mathrm{~mm}$, des Ecdyonurus de $2,5 \mathrm{~mm}$. Un A. crassipes de 8 à $9 \mathrm{~mm}$ peut vider complètement des Chironomini de $3 \mathrm{~mm}$ de longueur, dont le diamètre n'est égal qu'au double de celui de son appareil buccal.

D'une façon générale, le rendement au niveau de la prise de nourriture, est supérieur avec un prédateur nettement plus gros que sa proic.

\section{2) Temps de succion.}

Il esi variable :

- selon la nature de la proie. Les Plécoptères, les Tipulidae s. s. et les $A$. ibis tués par cannibalisme sont très souvent abandonnés prématurément ;

- selon la tailte de la proie.

Exemple 1: 1 Chironomus thummi thummi de $3 \mathrm{~mm}$ sucé complètement par un $A$. crassipes de $9 \mathrm{~mm}$ en $12 \mathrm{mn}$ à $18^{\circ} \mathrm{C}$.

Exemple 2: 3 Chironomus thummi thummi de 9 à $12 \mathrm{~mm}$ sucés à peu près complètement par $3 A$. crassipes de 8 à $9 \mathrm{~mm}$ en 63,106 et $116 \mathrm{mn}$ à $18^{\circ} \mathrm{C}$.

Exemple 3:4 Protonemura beatensis de $7 \mathrm{~mm}$ sucés incomplètement par $4 \mathrm{~A}$. ibis de 11 à $18 \mathrm{~mm}$ en $10,14,21$ et $46 \mathrm{mn}$ à $20^{\circ} \mathrm{C}$.

Exemple 4:7 Chironomus thummi thummi de 12 à $14 \mathrm{~mm}$ sucés à peu près complètement par des $A$. ibis de 11 à $18 \mathrm{~mm}$ : temps compris. entre 25 et $55 \mathrm{mn}$ à $20^{\circ} \mathrm{C}$.

Pour des animaux plus gros (Hexatoma ou Potamophylax de $15 \mathrm{~mm}$ ) le temps de succion peut largement dépasser une heure avec des $A$. ibis de $20 \mathrm{~mm}\left(12\right.$ à $\left.15^{\circ} \mathrm{C}\right)$.

Ces temps sont à rapprocher de ceux observés par Cameron (1934) sur des larves au $2^{\mathrm{e}}$ stade d'Haematopota pluvialis, nourris avec des Oligochètes, et compris entre 11 et $80 \mathrm{mn}$ avec un temps moyen de $37 \mathrm{mn}$.

\section{3) Nécrophagie.}

Après un jeûne prolongé, $A$. ibis peut consommer des cadavres frais, mais avec une réticence marquée. Si la mort remonte à plus de $12 \mathrm{~h}$, les chances de succion - et même de perforation sont à peu près nulles ainsi que le montre le tableau 2 . 


\begin{tabular}{r|r||r|r|r} 
Eau & 58 A.ibis & 58 A.ibis & $11 \mathrm{~A}$.ibis & \\
$21{ }^{\circ} \mathrm{C}$ & $11 \mathrm{a} 18 \mathrm{~mm}$ & $11 \mathrm{a} 18 \mathrm{~mm}$ & $19 \mathrm{a} 24 \mathrm{~mm}$ & \\
\hline $1 / 4 \mathrm{~h}$ & 2 & 13 & 5 & $2 \mathrm{~h}$ \\
\hline $1 / 2 \mathrm{~h}$ & 2 & 9 & 0 & $12 \mathrm{~h}$ \\
\hline $1 \mathrm{~h}$ & 0 & 1 & 0 & $24 \mathrm{~h}$ \\
\hline frais & 12 & 118 & 37 & frais \\
\hline
\end{tabular}

Tableav 2. - Consommations successives par A. ibis de larves de Protonemura beatensis de 7 à $8 \mathrm{~mm}$ tuées par une brève immersion dans de l'eau à $35^{\circ} \mathrm{C}$ depuis $1 / 4,1 / 2,1$ h (à gauche) et de Chironomus thummi thummi de 12 à $14 \mathrm{~mm}$ tuées à $45^{\circ} \mathrm{C}$ depuis 2,12 et $24 \mathrm{~h}$ (à droite).

\section{4) Modalités de la perforation.}

Cn ne sait rien des facteurs qui déclenchent la détente de la partic antérieure des Athericidac et provoquent ainsi la perforation de la proie.

L'olfaction joue probablement un grand rôle car la succion d'un Chironomidac ou d'un Éphéméroptère inertes, attire en quelques minutes des congénères venus d'une distance de plus de $5 \mathbf{c m}$. D'autre part, les réactions d'un $A$. ibis à l'affût sous une pierre sont très différentes selon qu'il lui est présenté un congénère ou par exemple un Nemouridae. Dans les deux cas, l'Atherix avance son extrémité antérieure en direction de la proie éventuelle, comme pour la reconnaître. Il la touche même souvent. Mais la détente, fulgurante, n'a lieu qu'en présence du Nemouridae. Elle est précédée d'un télescopage des segments antérieurs. L'amplitude de cette détente varie, selon l'éloignement de la proie, de moins d'un $\mathrm{mm}$ à plus de $5 \mathrm{~mm}$.

A. ibis est également capable de perforer au passage un Chironomide en nage rapide et paraît, même dans ce cas, ne jamais manquer son coup, contrairement à Haematopota pluvialis, Tabanidae beaucoup plus chitinisé (Cameron 1934).

La transmission dans l'eau des vibrations émises par la proie permet peut être aussi la localisation de celle-ci.

\section{5) Impact des larves d'A. ibis sur une population de Chironomidae.}

Le tableau 1 montre l'intensité, à un moment donné, de la prédation exercée par des $A$. ibis, séparés en quatre classes de taille, sur des larves de Chironomus thummi thummi de 10 à $13 \mathrm{~mm}$ offertes en large excès. Expérience effectuéc entre $23 \mathrm{~h}$ et $8 \mathrm{~h}$ du matin. Les Atherix avaient subi un jeûne de 12 jours depuis le prélèvement, cannibalisme excepté.

Le nombre $y$ de Chironomidae perforés par individu est une fonction linéaire de la longueur $x$ du prédateur ( $x$ étant exprimée ici en $\mathrm{mm}$ ) jusqu'à $15 \mathrm{~mm}$ environ (fig. 1 ).

$$
r=0,9971 \quad y=0,26 x-1,31 .
$$


Malgré le petit nombre de couples de données, le coefficient de corrélation $r$ est hautement significatif. La succion des proies est très inégale d'une classe à l'autre et croît nettement avec la taille de l'Athericidae. L'équation paraît donc traduire l'aptitude à la détente et à la perforation jusqu'à une longueur du prédateur au moins égale à celle de la proie, pour l'espèce choisie ici.

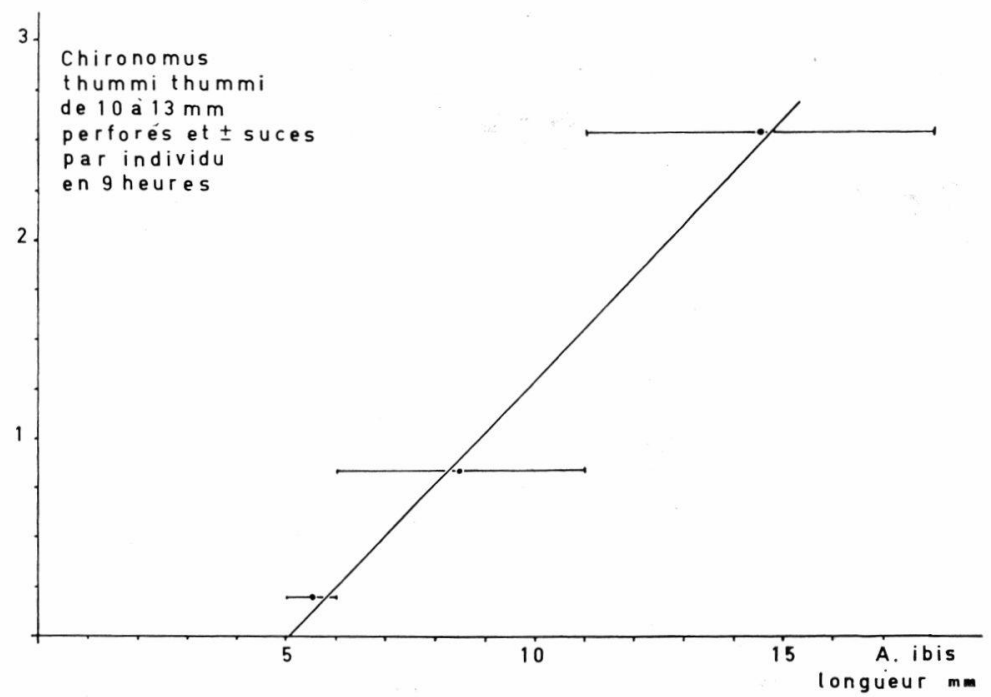

Fig. 1. - Nombres de Chironomus thummi thummi perforés par individu, et plus ou moins partiellement sucés, en une nuit. A. ibis à jeun depuis 12 jours $19-20^{\circ} \mathrm{C}$.

Le quatrième point, correspondant à une longueur moyenne de $19,8 \mathrm{~mm}$, est aligné avec les trois premiers mais sa position est incertaine à cause du petit nombre de larves d'A. ibis de cette classe de tailles (en particulier, l'une d'elles était mourante à la fin de l'expérience) et de la plus grande difficulté de vérifier si celles-ci avaient toutes participé à la prise de nourriture. Pour les tailles inférieures, il est en effet aisé de voir par transparence l'hémoglobine des Chironomidae dans le tube digestif des Atherix.

\section{V. - CONCLUSION}

Les larves d'Athericidae, et en particulier d'Atherix ibis, sont des carnivores stricts redoutables, se nourrissant à peu près exclusivement de larves d'autres insectes aquatiques. Toutefois, la diversité de leur régime alimentaire leur permet de s'adapter à de brutales diminutions de biomasse d'une proie préférentielle 

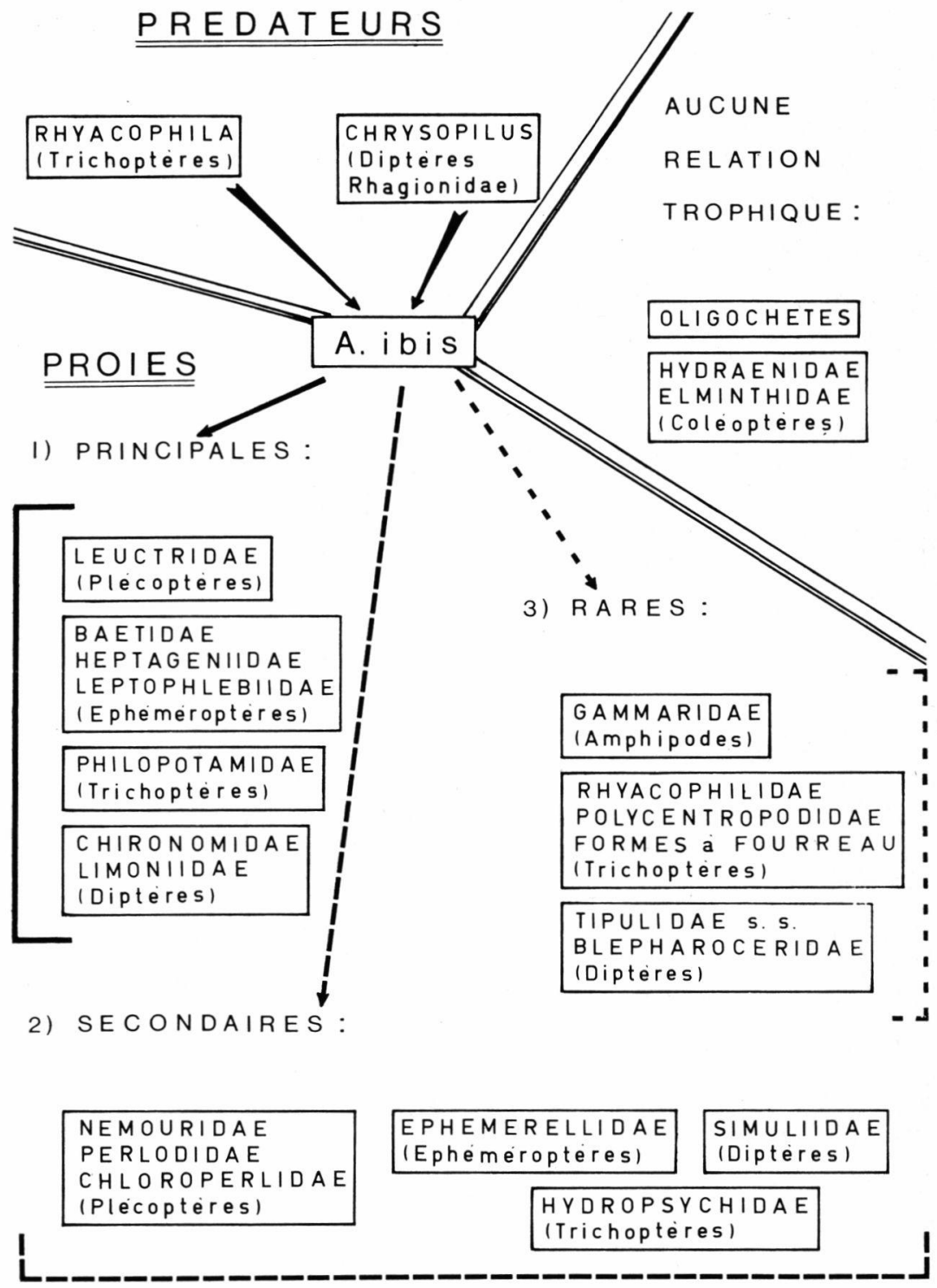

Tableau 3. - Principaux prédateurs des larves d'A. ibis de plus de $15 \mathrm{~mm}$. Proies de la même espèce sur fonds de pierres. 
(émergence massive d'un Éphéméroptère par exemple). A cette euryphagie s'ajoutent l'absence de comportement territorial net, l'absence pratique de cannibalisme dans des conditions normales et surtout une faculté de résistance prolongée au jeûne. Ces caractères constituent des adaptations efficaces à une surpopulation éventuelle et leur permettent de pulluler dans certains cours d'eau (Aldrich 1912, Thomas 1975).

Chez les proies, les facteurs limitant la prédation faite par les Athericidae sont l'épaisseur de la cuticule et probablement les sécrétions de mucus beaucoup plus que la vivacité, le régime alimentaire ou la taille par elle-même.

Enfin, la quantité de nourriture ingérée par les Athericidae par rapport au nombre de proies tuées paraît nettement supérieure à celle de beaucoup d'insectes torrenticoles prédateurs, en particulier lorsque ces derniers ne peuvent avaler leur proie toute entière.

\section{TRAVAUX CITES}

Aldrich (J. M.). 1912. - Flies of the Leptid genus Atherix used as food by California Indians (Dipt.). Ent. News, 23 (4) : 159-163.

Cameron (A. E.). 1934. - The life history and structure of Haematopota pluvialis Linné (Tabanidae). Trans. R. Soc. Edinb., 58 : 211-250.

Chvåla (M.), Lyneborg (I.) et Moucha (J.). 1972. - The Horse Flies of Europe (Diptera, Tabanidae). 500 p. + VIII pl. Copenhagen.

Hynes (H. B. N.). 1970. - The Ecology of Running waters. Liverpool University Press, 555 p.

IsaAC (P. V.). 1924. - Papers on Indian Tabanidae. A practical and simple method for rearing Tabanid larvae. Mem. Dep. Agric. India ent. Ser., $8: 53-57$.

Marchand (W.). 1917. - An improved method of rearing Tabanid larvae. J. econ. Ent. Concord, 10.

Nigatom (A.). 1962. - Studies in the aquatic Snipe flies of Japan. Part V. Biological notes. Mushi, 36 (10) : 103-149.

Schremmer (F.). 1951. - Die Mundteile der Brachycerenlarven und der Kopfbau der larve von Stratiomys chamaeleon L. öst. zool. $Z$., 3 (3-4) : 326-397.

Schward (H. H.). 1937. - Family Tabanidae. Methods for collecting and rearing Horse flies. In Culture Methods for invertebrate animals. Dover Publ., 590 p. New York.

Surcouf (J. M. R.). 1924. - Les Tabanides de France et des pays limitrophes. Lechevalier, 261 p., Paris.

TAchet (H.). 1975. - Recherches sur le comportement alimentaire et le comportement constructeur chez la larve de Plectrocnemia conspersa (Trichoptera, Polycentropodidae). Thèse de Doclorat d'Etat. Univ. Lyon 1, $\mathrm{n}^{\circ}$ 75-3.

Teskey (H. J.). 1969. - Larvae and pupae of some Eastern North American Tabanidae (Diptera). Mem. Ent. Soc. Can., 63 : 1-47. 
Thomas (A. G. B.). 1974 a. - Diptères torrenticoles peu connus : I. Les Athericidae (Larves et imagos) du Sud de la France (Brachycera, Orthorrhapha). Annls Limnol,, 10 (1) : 55-84.

Thomas (A. G. B.). 1974 b. - Diptères torrenticoles peu connus : II. Les Athericidae (nymphes) du Sud de la France (Brachycera, Orthorrhapha). Annls Limnol., 10 (2) : 121-130.

Thomas (A. G. B.). 1975. - Diptères torrenticoles peu connus : IV. Les Athericidae (Ecologie et Biologie) du Sud de la France (Brachycera, Orthorrapha), (sous presse).

Tsacas (L.). 1962. - Recherches sur la structure et le fonctionnement de la tête et des pièces buccales larvaires des Rhagionidae (Diptères). Mém. Mus. natn. Hist. nat., Paris, série A, 27 (2) : $147-235+16 \mathrm{pl}$.

VAILlant (F.). 1955. - Recherches sur la faune madicole (hygropétrique s.l.) de France, de Corse et d'Afrique du Nord. Mém. Mus. natn. Hist. nat., Paris, série A, $11: 1-252+6$ pl.

VAŇHARA (J.). 1975. - Larval development of Atherix marginata (Diptera, Athericidae) under natural conditions. Acta ent. bohemoslov., 72 (2) : 92-98.

Wyniger (R.). 1953. - Beiträge zur Oekologie, Biologie und Zucht einiger europäischer Tabaniden. Acta trop., 10 (4) : 310-347. 